largely evolved in many and varied ways, dependent on local need and interest.

I work for the Asylum Seeker and Refugee Mental Health Service in Plymouth, a specialist community team with a small staff complement, dedicated to the out-patient assessment and treatment of adults. If I had one wish for the next step in supporting this important group, it would be to see the union of grass-roots experience with a development of strategy and operational policy to support mainstream clinicians far and wide. The goal would be to build knowledge, skills, confidence, capacity, sustainability and leadership in delivering equality and meeting the diverse needs of this group.

This collection of articles begins a theme in the BJPsych International that is intended to continue across future editions. We will be hearing from those grass-roots. In this issue, we begin with a helpful editorial overview of some of the mental healthcare needs and considerations in meeting those needs, from the eyes of a UK-based consultant psychiatrist. We then move on to the current state of play for mental health services for asylum seekers and refugees in Malta and the Republic of Ireland. Our psychiatric colleagues succinctly paint pictures of the diverse structures of local health and community services and of the interfaces between agencies, and they give thoughtful consideration to what could work locally. Undoubtedly, there are resounding shared aspirations to see mental health service provision for asylum seekers and refugees improve and evolve.

We encourage College members to join the Asylum Seekers and Refugees Mental Health Network (see p. 51 of this issue), a space in which to share grass-roots clinical experience and develop our services together.
THEMATIC PAPER

\title{
The mental health needs of asylum seekers and refugees - challenges and solutions
}

\author{
Piyal Sen
}

Consultant Forensic Psychiatrist, Complex Needs Service, St Andrew's, Essex, UK; Visiting Lecturer, Department of Forensic and Neurodevelopmental Sciences, Institute of Psychiatry, Sciences, Institute of Psychiatry,
Psychology and Neuroscience, King's College London, UK, email psen@standrew.co.uk
Global events like wars and natural disasters have led to the refugee population reaching numbers not seen since the Second World War. Attitudes to asylum have hardened, with the potential to compromise the mental health needs of asylum seekers and refugees. The challenges in providing mental healthcare for asylum seekers and refugees include working with the uncertainties of immigration status and cultural differences. Ways to meet the challenges include cultural competency training, availability of interpreters and cultural brokers as well as appropriately adapting modes of therapy. Service delivery should support adjustment to life in a foreign country. Never has the need been greater for psychiatrists to play a leadership role in the area.

\section{Definitions}

A refugee is defined by the United Nations as:

A person who owing to a well-founded fear of being persecuted for reasons of race, religion, nationality, membership of a particular social group or political opinion, is outside the country of his nationality and is unable or, owing to such fear, is unwilling to avail himself of the protection of that country; or who, not having a nationality and being outside the country of his former habitual residence as a result of such events, is unable or, owing to such fear, is unwilling to return to it. (United Nations Convention Relating to the Status of Refugees, 1951, amended by the 1967 Protocol)

Asylum seekers are defined as people who have applied for asylum under the 1951 Refugee Convention on the ground that if they are returned to their country of origin they have a well-founded fear of persecution on account of race, religion, nationality, membership of a particular social group or political opinion. They remain asylum seekers for so long as their application or appeal against refusal of their application is pending. Asylum seekers should be distinguished from economic migrants, who choose to enter another country for paid employment.

\section{Challenges}

Because of global events like wars and natural disasters, but also because of increased freedom of travel, which is exploited by people-trafficking networks, the number of refugees has reached levels not seen in western Europe since the Second World War. According to figures from the end of 2014, there were nearly 20 million refugees throughout the world, and that number is now likely to be even greater (United Nations, 2016). 
At least $80 \%$ of all refugees are from low- and middle-income countries and half are children. There are great challenges in meeting their needs, because of the protracted nature of modern warfare, an increasingly dangerous climate for humanitarian workers and erosion of the concept of legitimate asylum. There are reported to be increasingly negative public attitudes in some highincome countries towards immigrants, including refugees and asylum seekers.

Asylum seekers are at particular risk of developing mental illness, including post-traumatic stress disorder (PTSD), depression and anxiety (Fazel et al, 2005). That risk is enhanced by their immigration status, time in detention, unemployment, absence of family support and complex asylum processes (Bhugra et al, 2014). Their needs pose significant challenges for mental health services.

What are the challenges in providing mental healthcare for this group? One of the greatest challenges for asylum seekers is coping with the uncertain nature of their immigration status and, in particular, the difficulties negotiating the process of seeking asylum. If their asylum application is refused, that could lead to destitution, and there is the associated threat of detention (Robjant et al, 2009).

A major challenge for mental health workers is understanding how different cultural groups communicate psychological distress. Lack of knowledge could lead to some patients receiving inappropriate psychiatric diagnoses, and normal human responses to extremely traumatic life events could be inappropriately pathologised. On the other hand, there is a risk of missing clinically significant disorders caused by trauma; psychological distress is commonly manifested in terms of somatic complaints in some cultures. Mental health workers need to understand that 'talking therapies' that have been developed in high-income countries are primarily ego-based and require detached introspection. Such introspection could be alien to a socio-centric individual, who might respond better to a treatment that is focused on functional recovery ('What do you need to do?') than an emotion-focused approach ('How are you feeling?') (Summerfield, 2001).

\section{Solutions}

The use of interpreters is important, as language difficulties are a significant barrier to effective consultation, and cultural brokers or cultural consultants could foster trust and help to improve understanding of the individual's life situation. The therapeutic team should be in a position to offer information about mental health resources, local refugee community organisations, and how to access legal support as well as information on employment, education and housing. It might be desirable for areas with large numbers of asylum seekers to develop a specialist team or specialists within a team.

Training in cultural awareness and cultural competency should be mandatory for all health professionals, to give them a set of academic and interpersonal skills that will allow them to manage diverse populations and to understand cultural differences and similarities. Types of psychotherapy that have an evidence base for their effectiveness in the management of at-risk populations include narrative exposure therapy (NET) and cognitivebehavioural therapy (CBT) (Patel et al, 2014). The model of therapy should be sufficiently flexible to incorporate patients' own belief systems and those of their families or carers.

An individual's response to pharmacotherapy will also vary, depending on biological factors. Some ethnic groups could respond to psychotropic medication in ways that are not predictable, depending on variables such as gender, enzymatic (genetic) and dietary differences. Attitudes towards medication and the potential for the patient's parallel use of complementary medication must also be borne in mind when prescribing for at-risk immigrants (Bhugra et al, 2014).

Maintaining close links with general medical services is crucial. The model of service delivery for refugees and asylum seekers will depend on the country in which it is offered. In low-income countries, where there is little access to specialist services, the focus should be more on building capacity (Patel et al, 2006), along with training of professional staff and consultation with voluntary organisations. In high-income countries, though there could be a hostile political climate, the same issues apply, although there is the additional need to adapt the usual model of service (Vostanis, 2014).

Better tools are needed to estimate the prevalence of mental disorders as well as to assess the needs of local refugee populations. Specialised models of service delivery are being developed for prevention and early intervention; communitybased approaches should be focused on increasing knowledge and awareness of mental health issues as well as supporting the process of adjusting to life in the host country (Nazzal et al, 2014). Children are at particular risk of receiving suboptimal mental healthcare for difficulties arising from pre-migration and post-migration stress, in part because of the impact of resettlement on their parents' ability to provide care. Service utilisation rates are low, and that could reflect the tendency of the host country's services to attribute their problems to social rather than psychological causes, as well as the family's lack of understanding, or distrust of mental health services (Colucci et al, 2014). Child and adolescent mental health services should introduce evidence-based programmes to support them.

Forensic professionals encounter asylum seekers or refugees when they commit a crime. If there are associated mental health problems, the person could be transferred to a secure unit. If patients have committed a crime they may be liable to automatic deportation under UK law, or patients may have exhausted appeal rights for an asylum claim. Their psychiatrist should make appropriate 
after-care arrangements before discharge, bearing in mind that there could be a threat of deportation to a country where psychiatric care is limited. Forensic services also come into contact with asylum seekers while offering in-reach services to prisons, where the proportion of foreign nationals is growing (currently about 13\%). They represent a particularly vulnerable section of the prison population, yet have low levels of contact with mental health services (Sen et al, 2014).

There is an urgent need for forensic psychiatrists to be involved in a proper mental health needs assessment of foreign nationals in prison, as well as those held in detention centres, to plan better services to meet their need. The prospect of indefinite detention and inadequate care could contribute to a deterioration of their mental health.

\section{Conclusion}

In the debate about refugees and asylum seekers, a drive towards inclusive globalisation is in conflict with a drive to restore the identity of the nation state, and exclusivity. Sigmund Freud was aware of these tensions nearly a century ago, and described them in Civilization and Its Discontents (1929). As mental health professionals, our task should be to understand and work with these tensions. Never has the need been greater for psychiatrists to play a leadership role in such a politically contentious and emotionally charged area.

\section{References}

Bhugra, D., Gupta, S., Schouler-Ocak, M., et al (2014) EPA guidance: mental health care of migrants. European Psychiatry, 29, 107-115.

Colucci, E., Szwarc, J., Minas, H., et al (2014) The utilisation of mental health services by children and young people from a refugee background: a systematic review. International Journal of Culture and Mental Health, 7, 86-108.

Fazel, M., Wheeler, J. \& Danesh, J. (2005) Prevalence of serious mental disorder in 7000 refugees resettled in Western countries: a systematic review. Lancet, 365, 1309-1314.

Nazzal, K. H., Forghany, M., Geevarughese, M. C., et al (2014) An innovative community-oriented approach to prevention and early intervention with refugees in the United States. Psychological Services, 11, 477-485.

Patel, N., Kellezi, B. \& Williams, A. C. D. C. (2014) Psychological, social and welfare interventions for psychological health and well-being of torture survivors. Cochrane Database of Systematic Reviews, 11, CD009317 (doi:10.1002/14651858.CD009317.pub2)

Patel, V., Boardman, J., Prince, M., et al (2006) Returning the debt: how rich countries can invest in mental health capacity in developing countries. World Psychiatry, 5, 67-70

Robjant, K., Hassan, R. \& Katona, C. (2009) Mental health implications of detaining asylum seekers: systematic review. British Journal of Psychiatry, 194, 306-312.

Sen, P., Exworthy, T. \& Forrester, A. (2014) Mental health care for foreign national prisoners in England and Wales. Journal of Mental Health, 23, 333-339.

Summerfield, D. (2001) Asylum-seekers, refugees and mental health services in the UK. Psychiatric Bulletin, 25, 161-163.

United Nations (2016) Refugees: Numbers (Resources for Speakers on Global Issues). United Nations. Available at http://www.un.org/ en/globalissues/briefingpapers/refugees/index.shtml (accessed 7 January 2016).

Vostanis, P. (2014) Meeting the mental health needs of refugees and asylum seekers. British Journal of Psychiatry, 204, 176-177.

\title{
THEMATIC PAPER
}

\section{The mental health services for detained asylum seekers in Malta}

\author{
Rachel Taylor-East,, ${ }^{1}$ Alexia Rossi, ${ }^{2}$ Julian Caruana ${ }^{3}$ and Anton Grech ${ }^{4}$
}

${ }^{1}$ Resident Specialist Psychiatrist, Mount Carmel Hospital and Community Mental Health Services, Malta; Visiting Assistant Lecturer, University of Malta, email rachel.taylor-east@gov.mt ${ }^{2}$ email rachel.taylor-east Counselling Psychologist, Jesuit Refugee Services, Malta: Senior Visiting (ecture, University of Malta ${ }^{3}$ Chartered Counselling Psychologist, Jesuit Refugee Services, Malta: Senior Visitin Lecturer, University of Malta Department of Health, Malta Resident Senior Lecturer in Psychiatry, University of Malta

\begin{abstract}
Approximately 17000 individuals have claimed asylum in Malta over the past 10 years. Maltese law stipulates mandatory detention. Here, we review Malta's asylum procedures and detention policy, and explore the impact of detention on mental health. We review the current mental health services and make recommendations to help fill the gaps.
\end{abstract}

\section{Malta's asylum procedures and detention policy}

Approximately 17000 individuals have claimed asylum in Malta over the past 10 years, with the majority having entered the country as unauthorised boat arrivals $-92.3 \%$ of asylum applicants between 2005 and 2014 according to the United Nations High Commissioner for Refugees
(UNHCR, 2015). The majority of these arrivals hail from sub-Saharan Africa, with more than half originating from Somalia and Eritrea. Between 2004 and 2014, 65.3\% of all asylum applicants were offered some form of protection (UNHCR, 2015).

Maltese law stipulates that every individual who enters, or is present in, Malta without authorisation is subject to a removal order that triggers mandatory detention. In practice this means that, although such individuals can apply for asylum, they will be detained while their application is being processed. If granted some form of protection they are immediately released from detention. According to Maltese immigration law, asylum seekers can be detained for a maximum of 12 months, while detainees whose application is rejected before the lapse of a year are detained for a maximum of 18 months. The only exemption to the regime of mandatory detention applies to 